\title{
Sistema Mitralign de Anuloplastia Mitral Percutânea para Pacientes com Regurgitação Mitral e Insuficiência Cardíaca Congestiva: Experiência First-In-Man
}

\author{
Santiago Felipe Gallo Merino' ${ }^{1}$ Adrian Ebner ${ }^{1}$, Eduardo Alvarez ${ }^{1}$, Enrique Silva ${ }^{1}$
}

\section{RESUMO}

Introdução: A regurgitação mitral não tratada resulta em aumento da mortalidade em pacientes com insuficiência cardíaca congestiva. O Sistema Mitralign de Anuloplastia Mitral Percutânea propicia uma opção de tratamento sem cirurgia em casos selecionados, com dilatação do anel mitral. Método: O procedimento é realizado por meio de uma única punção da artéria femoral com introdutor $14 \mathrm{~F}$, sendo usados para visualização tanto a fluoroscopia como o ecocardiograma transesofágico. O cateter-guia é manobrado até o ventrículo esquerdo e posicionado sob o anel da valva mitral. Um sistema de cateteres implanta fios de sutura no anel mitral posterior, que são então aproximados mediante um sistema de tração, remodelando o anel valvar. Uma vez alcançado o resultado desejado as suturas são fixadas e cortadas. Resultados: O procedimento foi realizado em 6 pacientes e foi bem tolerado. A duração do procedimento foi, em média, inferior a duas horas e, na maioria dos casos (4/6), houve redução imediata da regurgitação mitral. Todos os pacientes foram acompanhados por até 12 meses. Não ocorreu nenhum óbito, estando 4 pacientes em classe I, 1 paciente em classe II e 1 paciente em classe III da New York Heart Association. Obteve-se redução significativa dos diâmetros ventriculares esquerdos e também do tamanho do átrio esquerdo após os primeiros 90 dias de acompanhamento. Conclusão: Essa técnica pode ser realizada com segurança e mostra benefício considerável para pacientes selecionados, nos quais a cirurgia não é uma boa opção. Maior número de pacientes tratados com esse novo procedimento nos permitirá compreender melhor os limites da anuloplastia mitral percutânea.

DESCRITORES: Insuficiência cardíaca. Insuficiência da valva mitral/terapia. Cateterismo cardíaco.

1 Hospital Francês - Assunção, Paraguai.

Correspondência: Santiago Felipe Gallo Merino. Pedro de Mendoza e/Medicos del Chaco, 1321 - Pilar - Lambare, Paraguay

E-mail: santigallo56@yahoo.com

Recebido em: 10/4/2009 • Aceito em: 22/6/2009

\section{ABSTRACT}

Mitralign Percutaneous Mitral Annuloplasty System for Mitral Regurgitation in Congestive Heart Failure Patients: First-in-Man Experience

Background: Untreated mitral regurgitation results in increased mortality in patients with congestive heart failure (CHF). The Mitralign Percutaneous Annuloplasty System provides a treatment option without surgery in selected cases with mitral annulus dilation. Methods: The procedure is performed through a single $14 \mathrm{~F}$ femoral artery puncture; fluoroscopy and transesophageal echocardiography are both used for visualization. The guide catheter is maneuvered into the left ventricle and positioned at the posterior mitral annulus. A catheter system places sutured pledgets into the posterior mitral annulus and these implants are pulled together to remodel the mitral valve annulus. Once the desired effects are achieved, the suture is locked and cut. Results: The procedure was performed in 6 patients and was well tolerated. Procedure times were generally $<2$ hours and in most cases (4/6) immediate mitral regurgitation reduction was achieved. All patients were followed up to 12 months. There were no deaths and 4 patients were New York Heart Association class I, one was class II and one class III. There was a significant reduction of the left ventricular diameters, and left atrium size after the first 90 days of follow up. Conclusions: Direct percutaneous mitral annuloplasty is achievable and reproducible. This is a technique that can be performed safely and shows a considerable benefit for selected patients in whom surgery is not a good option. A higher number of patients treated with this new procedure will provide us with a better understanding of the limitations of percutaneous mitral annuloplasty approach.

DESCRIPTORS: Heart failure. Mitral valve insufficiency/ therapy. Heart catheterization.
A regurgitação da valva mitral aumenta o risco de mortalidade em pacientes com insuficiência cardíaca congestiva, doença que afeta mais de 25 milhões de pessoas no mundo inteiro. O risco de mortalidade após cinco anos, comparativamente a pacientes com insuficiência cardíaca congestiva sem re- 
gurgitação da valva mitral, aumenta em $18 \%$ com regurgitação da valva mitral leve e em 53\% com regurgitação da valva mitral moderada e grave. Os dados disponíveis demonstram que a correção da regurgitação da valva mitral deve ser feita antes da ocorrência de descompensação do ventrículo esquerdo e insuficiência cardíaca congestiva. A intervenção precoce com o objetivo de reconstruir a valva mitral pode gerar grande impacto sobre o progresso natural da doença. Rankin et al. ${ }^{1}$ relataram que os pacientes selecionados para reconstrução da valva habitualmente apresentam taxas de sobrevida maiores que aqueles cujas valvas têm que ser substituídas. A abordagem padrão é a cirurgia de coração aberto, mas ela só é realizada numa minoria de pacientes com regurgitação da valva mitral. Avanços recentes no conceito de reconstrução percutânea da valva mitral levaram a opções de tratamentos em estudo: 1. anuloplastia do seio coronário, que consiste em anuloplastia mitral parcial realizada por meio da colocação de um dispositivo no seio coronário para reduzir a circunferência do anel mitral posterior; e 2. reconstrução mitral com técnica edge-to-edge, em que os folhetos anterior e posterior são unidos utilizando-se um clipe ou sutura ${ }^{2-4}$. A anuloplastia do seio coronário enfrenta uma série de desafios, incluindo-se o potencial para comprometimento da artéria circunflexa esquerda ${ }^{5}$. O conceito de reconstrução mitral com a técnica edge-to-edge foi utilizado em pacientes tratados por cirurgia, mas os melhores resultados foram obtidos quando combinada à anuloplastia ${ }^{6}$.

O Sistema de Anuloplastia Percutânea Mitralign é uma nova opção de tratamento para pacientes selecionados que apresentaram dilatação do anel mitral e morfologia normal do folheto. O objetivo primário deste estudo é testar a viabilidade e a segurança para realizar a plicatura do anel mitral posterior com o Sistema de Anuloplastia Percutânea Mitralign em pacientes elegíveis com regurgitação da valva mitral funcional crônica, utilizando abordagem endovascular.

\section{MÉTODO}

Este é um estudo de coorte, first-in-man, conduzido no Hospital Francês, em Assunção, Paraguai, desde janeiro de 2008. Foram incluídos pacientes com regurgitação da valva mitral funcional crônica classe II-IV da New York Heart Association (NYHA), que tinham regurgitação da valva mitral grau $\geq 2+$, fração de ejeção do ventrículo esquerdo $\geq 25 \%$ e volume diastólico final do ventrículo esquerdo $\geq 55 \mathrm{~mm}$. Os critérios de exclusão considerados foram: pacientes com calcificação do anel mitral; estenose mitral ou aórtica limitando a possibilidade de implante do dispositivo; trombo no apêndice do átrio esquerdo; reconstrução prévia da valva mitral (por exemplo, presença de uma válvula biológica mitral ou anel de anuloplastia mitral preexistente); doença vascular periférica significativa impedindo o acesso percutâneo de uma bainha do introdutor de 14 F; uma condição médica, além de insuficiência cardíaca, que pudesse impedir a sobrevida em até 12 meses após o procedimento; pacientes com diátese hemorrágica ou hipercoagulação documentada; alergia conhecida a aço inoxidável, irídio, platina, poliéster ou meio de contraste; mulheres grávidas ou amamentando; pacientes incapazes de tomar anticoagulantes ou antiplaquetários; e pacientes que estivessem participando de estudo com medicamentos ou novos dispositivos.

Esse procedimento foi realizado sob sedação profunda ou anestesia geral em pacientes com sintomas instáveis. Todos os pacientes receberam injeções de heparina intravenosa para manter o tempo de coagulação ativado acima de 250 segundos e antibioticoterapia profilática. Após o procedimento, os pacientes receberam clopidogrel $75 \mathrm{mg} /$ dia e aspirina $325 \mathrm{mg} /$ dia durante os três primeiros meses.

\section{Detalhes do procedimento}

Utilizando fluoroscopia e ecocardiograma transesofágico para visualização, o procedimento foi realizado com um único introdutor arterial $14 \mathrm{~F}$, previamente acoplado a instrumental para dupla sutura ao final do procedimento. Um cateter $7 \mathrm{~F}$ foi utilizado para auxiliar a colocação do cateter-guia Mitralign no ventrículo esquerdo. O cateter-guia Mitralign 12,5 F foi desenhado para direcionar cateteres para o ponto central (P2) do anel mitral posterior (Figura 1). Uma vez atingida a posição P2, abaixo do folheto posterior, era colocado cateter retrátil com lúmen, através do qual era possível avançar um crossing wire até essa posição.

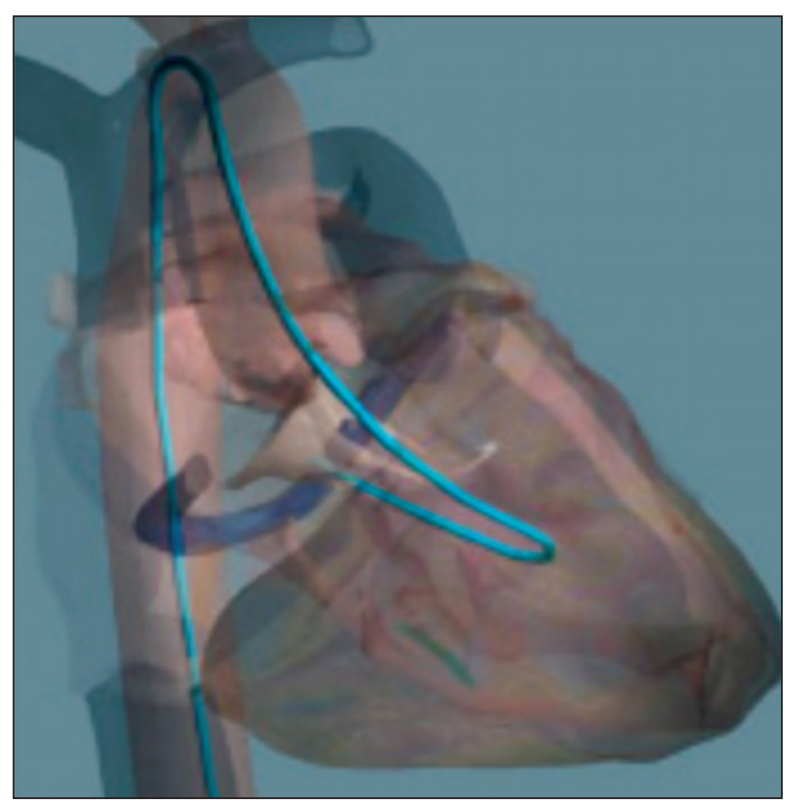

Figura 1 - Cateter-guia de 12,5 F proporciona acesso ao ventrículo esquerdo para todos os outros cateteres do sistema Mitralign. 
O crossing wire é um fio-guia de 0,018 polegada, com $3 \mathrm{~m}$ de comprimento, que, conectado a uma fonte de energia por radiofrequência, é utilizado para transfixar o anel do lado ventricular para o atrial, proporcionando uma via para o cateter de liberação de pledgets (implantes de poliéster que servirão de reforço para suturas). Após a colocação do primeiro crossing wire em P2, introduz-se o cateter Trident. O Trident é um cateter com triplo lúmen, desenhado para avançar sobre o crossing wire em $\mathrm{P} 2$, para permitir o posicionamento dos demais crossing wires nas posições laterais P1 e P3. Uma vez avançado até o anel, o cateter se abre em configuração de tridente, com os braços externos expandindo nas laterais em direção aos pontos P1 e P3, abrangendo, no total, uma extensão de $3 \mathrm{~cm}$ (Figura 2).

A próxima etapa é a liberação dos três pledgets com cateter $7 \mathrm{~F}$, desenhado para deslizar sobre o crossing wire e transfixar o anel mitral. Esse cateter libera a porção distal do pledget no lado atrial do anel e é então retraído até o lado ventricular, liberando a porção proximal dessa estrutura. Esses pledgets funcionam como reforço para as suturas que farão a plicatura do anel. O pledget incorpora dois marcadores radiopacos de platina-irídio que auxiliam sua liberação e, posteriormente, ajudam a avaliar a plicatura durante o procedimento. Quando os pledgets são liberados, um marcador $(4 \mathrm{~mm})$ fica visível no lado atrial da valva mitral e o outro $(1 \mathrm{~mm})$ fica visível no lado ventricular. Nesse momento, utiliza-se um mecanismo externo para auxiliar a amarração desses pledgets. Esse dispositivo tem um indicador visual que confirma se foi aplicada força suficiente para amarrar os pledgets (Figura 3).

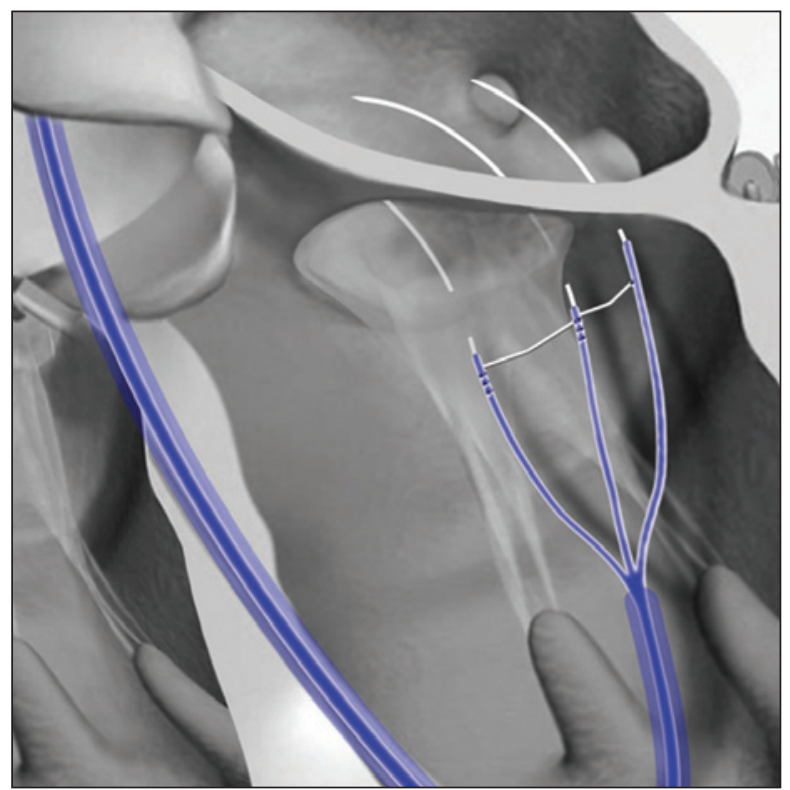

Figura 2 - O cateter Trident avançou sobre o crossing wire na posição P2 para fazer o posicionamento de P1 e P3. Os crossing wires penetram o anel no sentido do lado ventricular para o atrial.

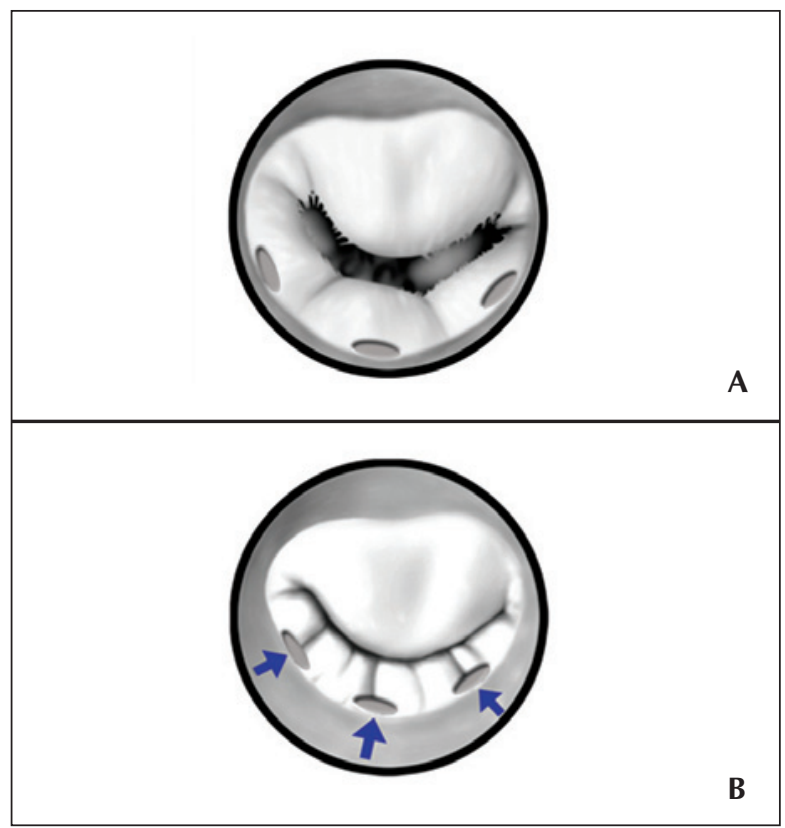

Figura 3 - Aspecto da valva mitral antes (A) e após (B) a plicatura.

A próxima etapa é realizada com cateter $10 \mathrm{~F}$ com lúmen central, pré-carregado com um implante lock (fechadura), que avança sobre as suturas dos pledgets até o anel. Uma vez avançado até o anel, o cateter de plicatura, juntamente com uma manopla externa (dispositivo para auxílio da plicatura), dá início à plicatura (Figura 4).

Uma vez obtida a plicatura, o cateter de plicatura e o dispositivo para auxílio da plicatura são utilizados para liberar o lock nas suturas, garantindo a plicatura. A liberação da tensão nas suturas antes de serem travadas mostrará, no ecocardiograma transesofágico, o impacto funcional da plicatura da parte posterior do anel mitral (Figura 5).

O suture cutter é um cateter $7 \mathrm{~F}$ que é avançado sobre as suturas, abaixo do anel posterior. Uma vez que esteja em posição proximal ao lock posicionado anteriormente, o operador ativa sua lâmina, permitindo que as suturas sejam cortadas. O introdutor é então retirado e as suturas da via de acesso são amarradas, encerrando o procedimento.

\section{RESULTADOS}

O procedimento foi bem tolerado pelos pacientes, com duração média de 145 minutos (95225 minutos). A média de idades foi de 47 anos (24-58 anos), sendo 4 pacientes do sexo masculino. As características basais são apresentadas na Tabela 1.

Os tempos de procedimento foram inferiores a duas horas e, na maioria dos casos (4/6), obteve-se 
Merino SFG, et al. Sistema Mitralign de Anuloplastia Mitral Percutânea para Pacientes com Regurgitação Mitral e Insuficiência Cardíaca Congestiva: Experiência First-In-Man. Rev Bras Cardiol Invas. 2009;17(2):183-9.

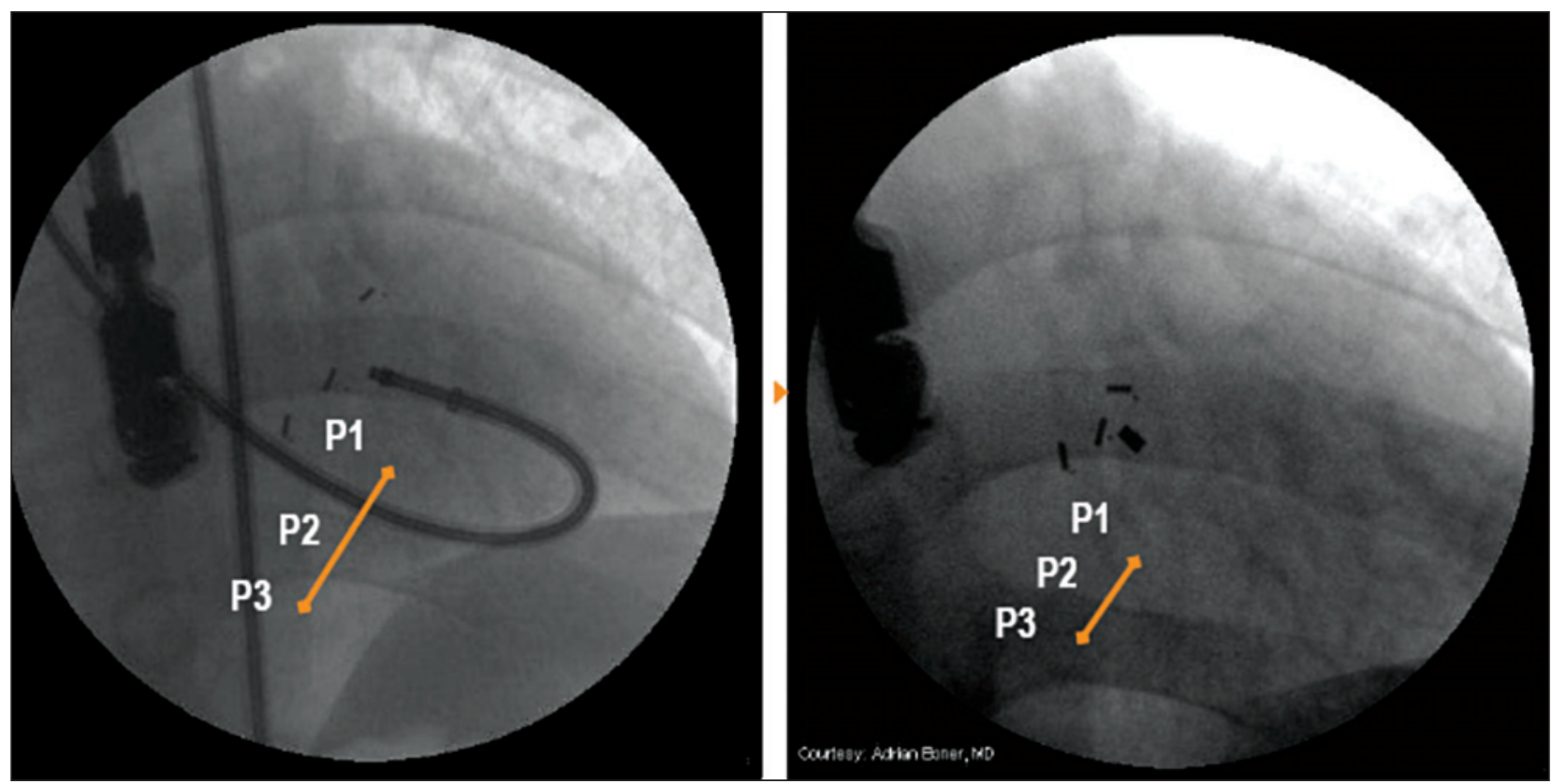

Figura 4 - Imagens de fluoroscopia mostrando as distâncias entre os pledgets antes (à esquerda) e após (à direita) o procedimento. O anel posterior é reduzido em $1,5 \mathrm{~cm}$ (redução de $50 \%$ da distância P1-P3, de $3 \mathrm{~cm}$ para $1,5 \mathrm{~cm}$ ).

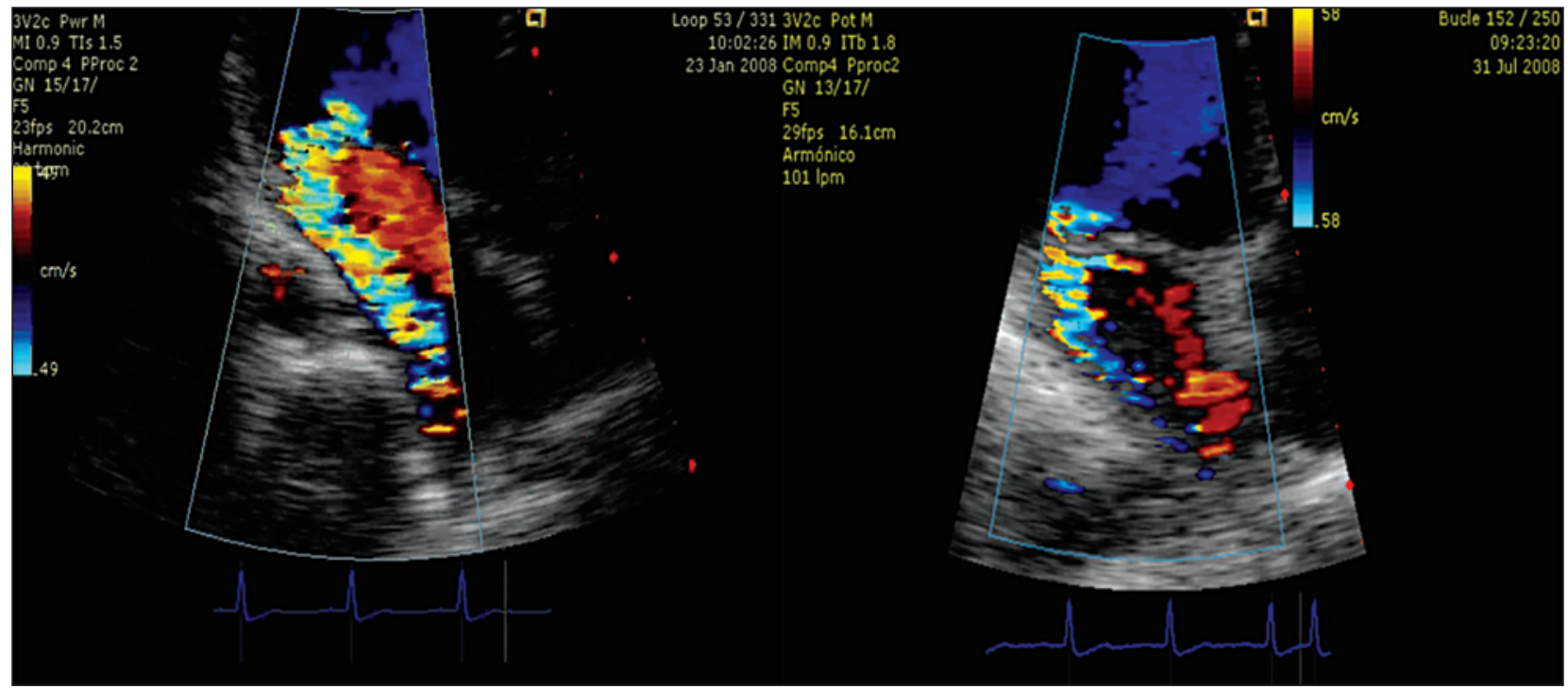

Figura 5 - Imagens do ecocardiograma transesofágico: à esquerda, insuficiência mitral grau 3 e à direita, grau 2 logo após o procedimento.

redução imediata da regurgitação mitral. Todos os pacientes foram acompanhados por até 12 meses. Não houve mortes e a maioria dos pacientes encontravase em classe I da NYHA (Tabela 2).

Reduções dos diâmetros do ventrículo esquerdo, assim como das dimensões do átrio esquerdo, foram observadas após os primeiros 90 dias de acompanhamento (Figura 6). Os parâmetros ecocardiográficos após 12 meses (volume diastólico final, volume sistólico final, área da valva mitral e coaptação da valva mitral) apresentaram resultados melhores de forma consistente (Figura 7).

\section{DISCUSSÃO}

As publicações iniciais sobre anuloplastia por sutura cirúrgica apresentaram resultados encorajadores, com taxas de morbidade e mortalidade inferiores às esperadas ${ }^{7}$. Estudos comparativos iniciais nos centros que faziam anuloplastia mitral tanto por sutura como por anel não observaram diferenças nas taxas de mor- 
TABELA 1

Características basais

\begin{tabular}{lc}
\hline Variável & $\mathbf{n = 6}$ \\
\hline Média de idade & 47 anos \\
& $(24-58$ anos $)$ \\
Sexo masculino & 4 \\
Hipertensão & 3 \\
Diabetes melito & 2 \\
Tratamento farmacológico & \\
Carvedilol & 6 \\
Furosemida & 6 \\
Espironolactona & 6 \\
Inibidores da ECA & 6 \\
Digoxina & 4 \\
Classe funcional segundo a NYHA & \\
III & 4 \\
IV & 2 \\
Regurgitação mitral & \\
$3+$ & 6 \\
\hline
\end{tabular}

$\mathrm{ECA}=$ enzima conversora da angiotensina; $\mathrm{n}=$ número de pacientes; NYHA $=$ New York Heart Association.

TABELA 2

Classe funcional de acordo com a

NYHA e avaliação ecocardiográfica

da regurgitação mitral durante o período de acompanhamento de 12 meses

\begin{tabular}{lcccccc}
\hline & \multicolumn{2}{c}{$\begin{array}{c}\text { Classe funcional } \\
\text { (NYHA) }\end{array}$} & & \multicolumn{2}{c}{$\begin{array}{c}\text { Regurgitação } \\
\text { mitral }\end{array}$} \\
\cline { 2 - 3 } \cline { 5 - 6 } & Antes & Depois & & Antes & Depois \\
\hline Paciente 1 & IV & II & & $3+$ & $3+$ \\
Paciente 2 & III & I & & $3+$ & $2+$ \\
Paciente 3 & IV & III & & $3+$ & $3+$ \\
Paciente 4 & III & I & & $3+$ & $2+$ \\
Paciente 5 & III & I & & $3+$ & $2+$ \\
Paciente 6 & III & I & & $3+$ & $2+$ \\
\hline NYHA $=$ New & \multicolumn{3}{l}{ York Heart Association. } \\
\hline
\end{tabular}

bidade ou mortalidade ou na classificação da NYHA após um ano.

Em estudos longitudinais mais recentes, Aybek et al. ${ }^{8}$ e Nagy et al. ${ }^{9}$ trataram 222 e 130 pacientes, respectivamente, com anuloplastia por sutura. Aybek et al. ${ }^{8}$ revelaram desempenho hemodinâmico satisfatório e melhora clínica, com 95,1\% dos pacientes livres de reoperação e sobrevivência de $87,2 \%$, aos 77 meses. Nagy et al. ${ }^{9}$ descreveram taxa atuarial livre de reoperação de $88,2 \%$ em um período de acompanhamento de

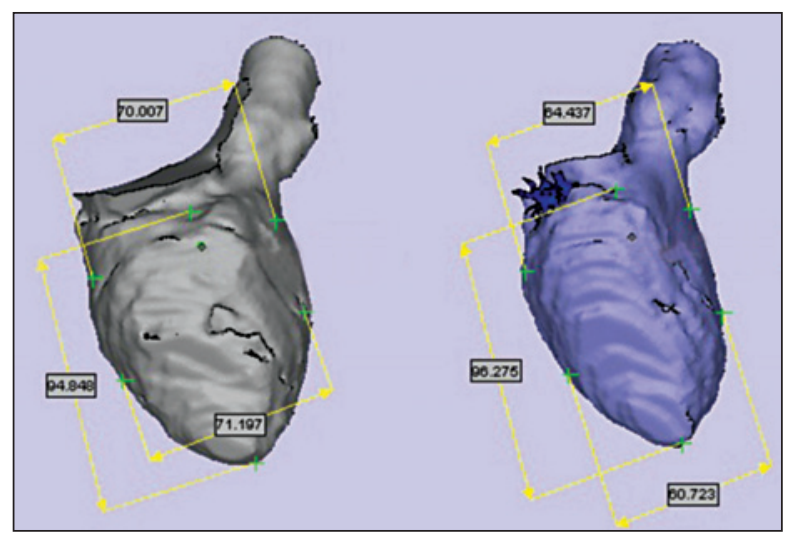

Figura 6 - Diâmetro ventricular esquerdo basal (à esquerda) e após 12 meses de acompanhamento (à direita), obtido por tomografia multislice (paciente 1), apresentando menores dimensões após a intervenção.

cinco anos. Ambos os estudos concluíram que o uso de materiais de sutura mais avançados (por exemplo, suturas mais fortes e não-expansíveis) otimizaria os resultados.

Por outro lado, Rankin et al. ${ }^{1}$ analisaram os dados de mais de 400 mil pacientes submetidos a procedimentos valvares cirúrgicos entre 1994 e 2003 e observaram que a cirurgia aberta para reconstrução de valvas, mesmo a reconstrução ou substituição exclusiva da valva mitral, está associada a risco de mortalidade não ajustado de $7,7 \%$. Essa taxa aumenta à medida que são acrescentadas outras comorbidades/variáveis, embora o efeito de uma única comorbidade seja modesto. Morbidades múltiplas ou específicas (como insuficiência renal) podem produzir efeitos significativos. As variáveis identificadas na literatura atual que afetam a taxa de mortalidade são: 1) apresentação aguda (vs. eletiva); 2) idade superior a 70 anos; 3 ) reoperação; 4) endocardite; 5) presença de insuficiência cardíaca congestiva; 6) sexo feminino; e 7) fração de ejeção do ventrículo esquerdo $<35 \%$. A adição de múltiplas comorbidades pode potencialmente aumentar a taxa de mortalidade da reconstrução da valva mitral para até $10,6 \%{ }^{1}$.

Vários dispositivos foram estudados e desenvolvidos para o tratamento percutâneo de pacientes com regurgitação mitral. Feldman et al. ${ }^{10}$ apresentaram, recentemente, no estudo Efficacy of Vasopressin Antagonism in Heart Failure Outcome Study with Tolvaptan (EVEREST), resultados de médio prazo de pacientes com regurgitação mitral tratados com reconstrução percutânea da valva mitral com a técnica edge-to-edge. A sobrevida de 36 meses, a sobrevida livre de cirurgia e a sobrevida livre de morte, cirurgia e regurgitação da valva mitral $>2+$ foram de $96 \%, 82 \%$ e $67 \%$, respectivamente. É importante ter em mente que essa abordagem é mais adequada para a regurgitação da valva mitral com má coaptação dos folhetos A2/P2 (regurgitação da valva mitral tipo II). 

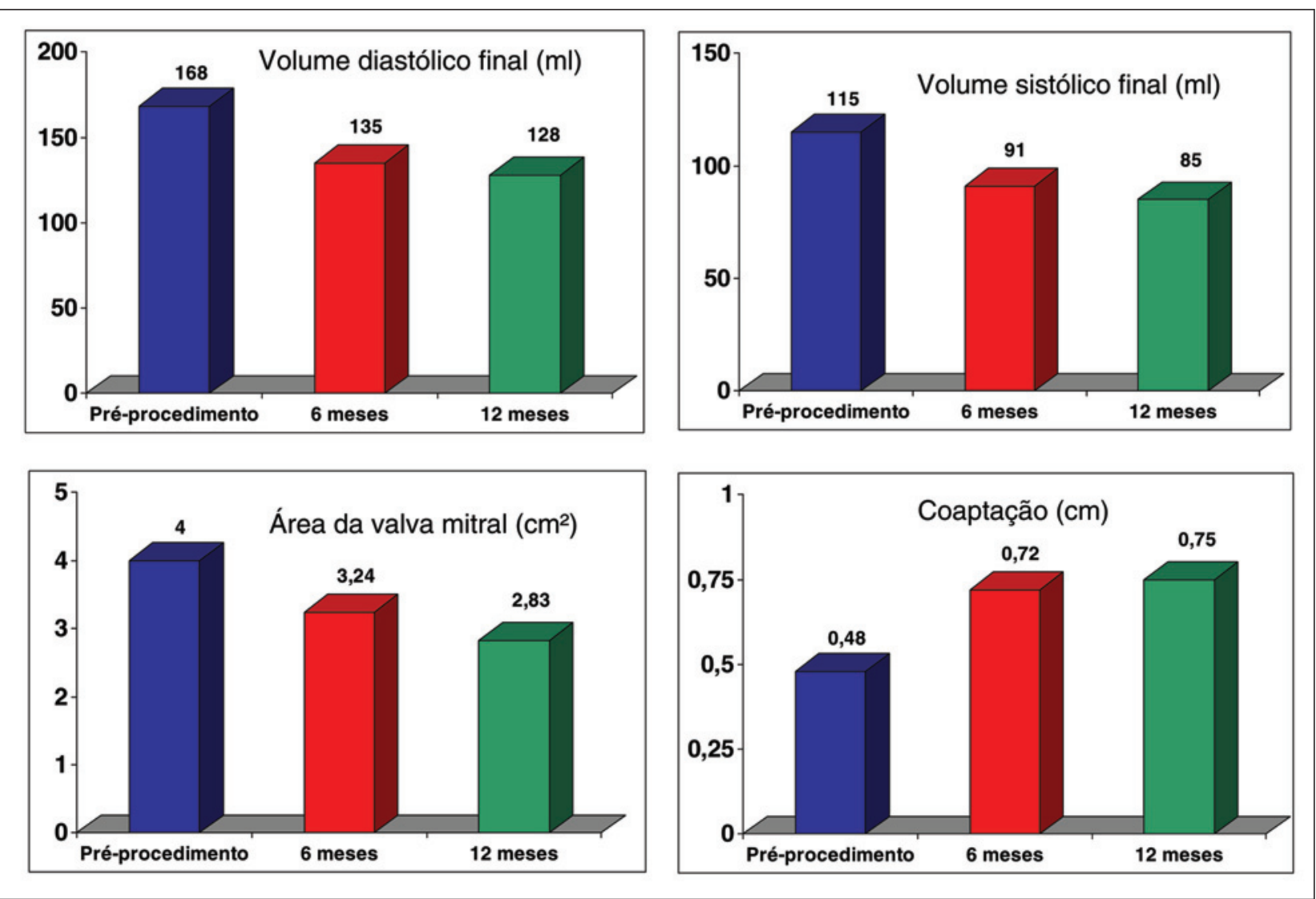

Figura 7 - Resultados ecocardiográficos mantidos após 12 meses.

As alterações na classe funcional da NYHA em nossos pacientes após 12 meses de acompanhamento caíram, na maioria dos casos, de classe III/IV para classe I da NYHA. Esse é um parâmetro de eficácia associado aos achados de ecocardiograma transesofágico, com redução da regurgitação da valva mitral de $3+$ para $2+$ imediatamente após o procedimento, que foi mantida durante a evolução. A redução de $50 \%$ da distância de P1-P3 (de $3 \mathrm{~cm}$ para 1,5 cm) foi essencial para reduzir a regurgitação da valva mitral, imitando o efeito da cirurgia descrito por Tibayan et al. ${ }^{11}$, em que a redução do diâmetro do anel posterior é eficiente quando a redução atinge no mínimo 20\% da dimensão septolateral.

Outros estudos descreveram o uso de dispositivos para realizar anuloplastia mitral percutânea através do seio coronário. Um grupo canadense ${ }^{12}$ publicou os resultados de pacientes tratados com anuloplastia mitral transvenosa percutânea (PTMA ${ }^{\mathrm{TM}}$ Viacor, Viacor, Inc., Wilmington, Estados Unidos). Após colocação e ajuste do dispositivo, o volume regurgitante foi substancialmente reduzido $(45,5 \pm 24,4 \mathrm{ml}$ para 13,3 \pm 7,3 ml) por meio da diminuição do diâmetro anterior-posterior do anel mitral $(40,75 \pm 4,3 \mathrm{~mm}$ para 35,2 \pm 1,6 mm) em 3 pacientes. Em 1 paciente, o dispositivo para anuloplastia mitral transvenosa percutânea não pôde ser liberado em decorrência da anatomia extremamente angulada. Esses autores concluíram que a anuloplastia mitral transvenosa percutânea é viável em seres humanos e reduz a regurgitação da valva mitral isquêmica por meio da redução do diâmetro anterior-posterior do anel mitral.

Resultados semelhantes foram obtidos por Duffy et al. ${ }^{13}$, que utilizaram um dispositivo distinto (Cardiac Dimension Temporary). A liberação do dispositivo resultou em redução significativa da dimensão do anel mitral septal-lateral de 35,5 \pm 4,7 $\mathrm{mm}$ para 32,2 \pm 4,6 $\mathrm{mm}$ $(P=0,02)$, com evidência de redução da área de regurgitação da valva mitral, ao Doppler colorido, de $98,3 \pm 43,6 \mathrm{~mm}^{2}$ para 83,3 $\pm 35,1 \mathrm{~mm}^{2}(P=0,09)$.

Os dados iniciais obtidos neste estudo, combinados com os dados pré-clínicos, proporcionarão importantes informações sobre os resultados técnico e clínico iniciais, que levarão à próxima fase de pesquisa clínica e potencialmente melhorarão o desenho futuro do Sistema de Anuloplastia Percutânea Mitralign.

\section{CONCLUSÃO}

A anuloplastia mitral direta percutânea é viável e reprodutível. Essa técnica pode ser realizada com se- 
gurança e apresenta benefício considerável para pacientes selecionados, nos quais a cirurgia não é uma boa opção. Maior número de pacientes tratados proporcionará melhor compreensão quanto aos limites da anuloplastia mitral percutânea.

\section{CONFLITO DE INTERESSES}

Os autores declararam inexistência de conflito de interesses.

\section{REFERÊNCIAS BIBLIOGRÁFICAS}

1. Rankin JS, Hammill BG, Ferguson TB Jr, Glower DD, O'Brien SM, DeLong ER, et al. Determinants of operative mortality in valvular heart surgery. J Thorac Cardiovasc Surg. 2006;131(3): 547-57.

2. Fucci C, Sandrelli L, Pardini A, Torracca L, Ferrari M, Alfieri O. Improved results with mitral valve repair using new surgical techniques. Eur J Cardiothorac Surg. 1995;9(11):621-6.

3. Maisano F, Torracca L, Oppizzi M, Stefano PL, D'Addario G, La Canna G, et al. The edge-to-edge technique: a simplified method to correct mitral insufficiency. Eur J Cardiothorac Surg. 1998;13(3):240-5.

4. Maisano F, Schreuder JJ, Oppizzi M, Fiorani B, Fino C, Alfieri $\mathrm{O}$. The double-orifice technique as a standardized approach to treat mitral regurgitation due to severe myxomatous disease: surgical technique. Eur J Cardiothorac Surg. 2000;17(3):201-5.

5. Lee MS, Shah AP, Dang N, Berman D, Forrester J, Shah PK, et al. Coronary sinus is dilated and outwardly displaced in patients with mitral regurgitation: quantitative angiographic analysis. Catheter Cardiovasc Interv. 2006;67(3):490-4.
6. Bhudia SK, McCarthy PM, Smedira NG, Lam BK, Rajeswaran J, Blackstone EH. Edge-to-edge (Alfieri) mitral repair: results in diverse clinical setting. Ann Thorac Surg. 2004;77(5): 1598-606.

7. Burr LH, Krayenbühl C, Sutton MS. The mitral plication suture: a new technique of mitral valve repair. J Thorac Cardiovasc Surg. 1977;73(4):589-95.

8. Aybek T, Risteski P, Miskovic A, Simon A, Dogan S, AbdelRahman $U$, et al. Seven years' experience with suture annuloplasty for mitral valve repair. J Thorac Cardiovasc Surg. 2006;131(1): 99-106.

9. Nagy ZL, Bodi A, Vaszily M, Szerafin T, Horvath A, Peterffy A. Five-year experience with a suture annuloplasty for mitral repair. Scand Cardiovasc J. 2000;34(5):528-32.

10. Feldman T. Mitral leaflet repair with the MitraClip I: trial methodology and results update from the EVEREST randomized clinical trial. In: Transcatheter Cardiovascular Therapeutics (TCT). Washington, October 2008. Disponível em: www.tctmd.com

11. Tibayan FA, Rodriguez F, Liang D, Daughters GT, Ingels NB Jr, Miller DC. Paneth suture annuloplasty abolishes acute ischemic mitral regurgitation but preserves annular and leaflet dynamics. Circulation. 2003;108 Suppl 1:I1128-33.

12. Dubreuil O, Basmadjian A, Ducharme A, Thibault B, Crepeau J, lam JY, et al. Percutaneous mitral valve annuloplasty for ischemic mitral regurgitation: first in man experience with a temporary implant. Catheter Cardiovasc Interv. 2007;69(7): 1053-61.

13. Duffy SJ, Federman J, Farrington C, Reuter DG, Richardson M, Kaye DM. Feasibility and short-term efficacy of percutaneous mitral annular reduction for the therapy of functional mitral regurgitation in patients with heart failure. Catheter Cardiovasc Interv. 2006;68(2):205-10. 